\title{
How to get ahead in imaging
}

\section{Advances in magnetic resonance imaging are helping scientists learn more about the structure and function of the brain. Nathan Blow looks at how far the technology has developed and where it could go.}

A wave of enthusiasm seems to overcome Joy Hirsch, a neuroscientist at Columbia University in New York, when she talks about recent developments in functional neuroscience. "I just love the new area of neuroeconomics," she says. Although not her primary field of study - her group focuses on how the brain uses cognitive control in decision-making processes - she says that understanding the neurophysiology behind financial decisions could make it possible to one day predict a person's financial habits by imaging the neural activity in their brain. And it is advances in cognitive research such as this that could eventually change the way we think about the brain-mind interface, says Hirsch.

The emergence of these areas in functional neuroscience can be traced in part to recent developments in brain imaging technology. "Magnetic resonance imaging used to be only in the hands of radiologists, but today more and more cognitive neuroscientists are using it," says Alan Koretsky, scientific director of the National Institute of Neurological Disorders and Stroke at the National Institutes of Health (NIH) in Bethesda, Maryland. And by taking advantage of magnetic resonance imaging (MRI), sometimes in combination with other methods such as electroencephalography (EEG) or positron emission tomography (PET), neuroscientists are exploring the structure of the human brain in greater detail than ever before and identifying specific regions that are active when a person is making a decision or feeling an emotion such as fear or happiness.

\section{Size is important}

With its high sensitivity and non-invasive nature, MRI is at the core of neuroimaging today. To create detailed anatomical and functional images, MRI systems take advantage of the ability of very large cylindrical magnets, ranging in strength from 1.5 to 15 tesla, to align the protons found in water throughout the body. Smaller, localized radio-frequency electromagnetic fields are then generated to push those protons out of alignment. The displaced protons generate signals that are detected by the MRI instrument and translated into an image.

Whether built for imaging humans or small animals, MRI instruments are defined by the field strengths of their magnets - and here bigger is better. The signal from magnetic resonance is inherently weak and it can be
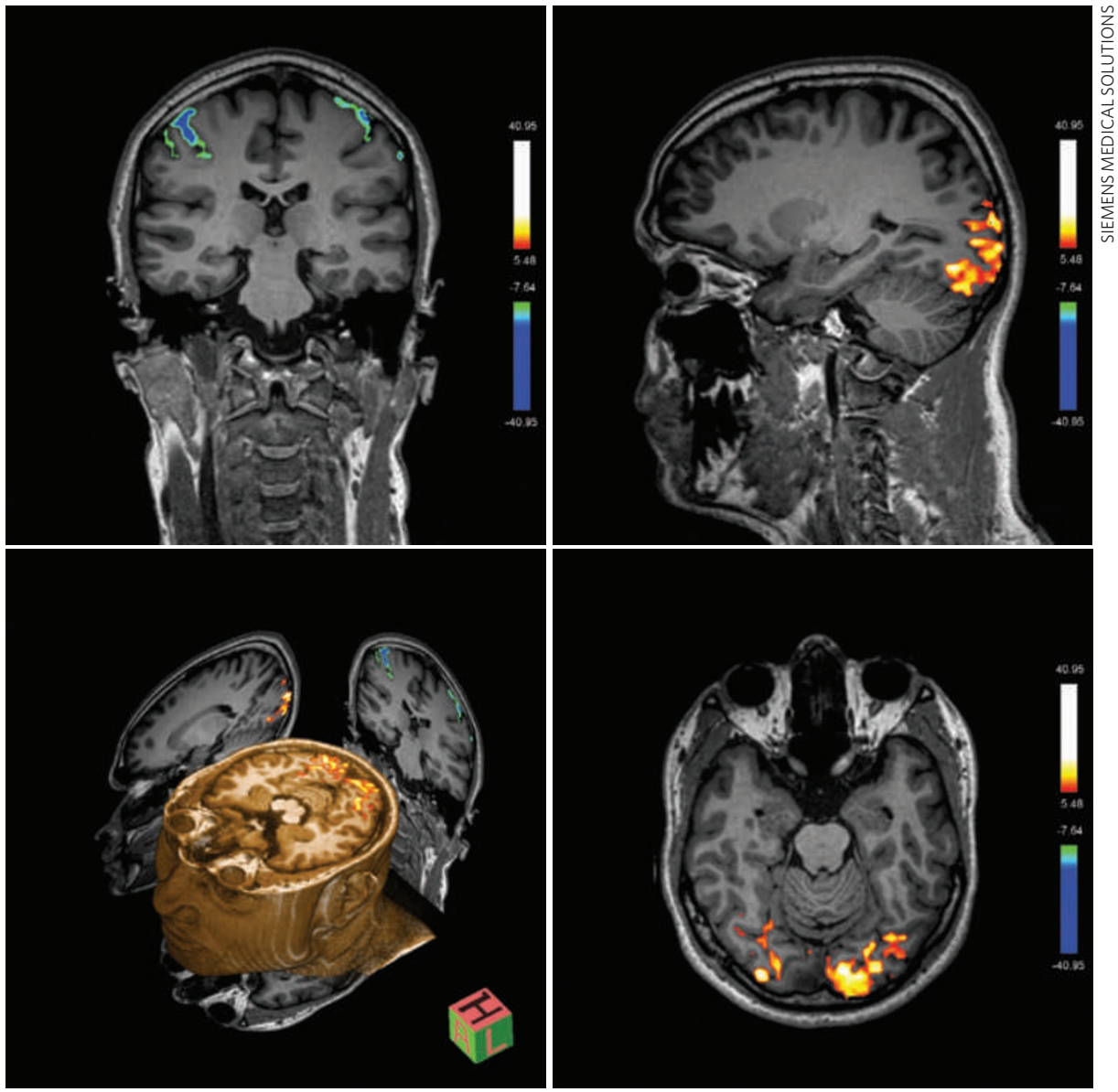

Magnetic resonance images, such as these taken with a 7-tesla scanner, are getting more and more detailed.

difficult to detect, which tends to limit resolution. So developers are constantly trying to increase the field strengths of their magnets and boost the signal.

"Small-animal imaging is based on making systems with magnets of smaller diameter but much higher field strength than you would get in clinical practice," says Roy Gordon, vice-president of imaging at Bruker Biospin, a scientific-instrumentation company in

"We would love an anatomical picture that looks like a histological slice." - Alan Koretsky Billerica, Massachusetts. Most animal-imaging magnets range in field strength from $4.7 \mathrm{~T}$ to $15 \mathrm{~T}$, with $7 \mathrm{~T}$ being the "workhorse field strength in animal imaging", according to Gordon. Human MRI instruments, on the other hand, use 0.5-3 T magnets

for clinical applications and up to $9.4 \mathrm{~T}$ for research applications.

Part of the challenge in moving human scanners up in field strength to match their small-animal counterparts comes from our body sizes. "Maintaining field strength homogenously over a large volume becomes more and more challenging as the field strength increases," says Vibhas Deshpande, a research and development scientist at Siemens Medical Solutions in Malvern, Pennsylvania. For this reason, he says, the human scanners that are operating at ultra-high field strengths are mostly using smaller diameter magnets, similar to animal scanners, to image small human samples such as tissues rather than performing whole-body scans.

\section{Image artefacts}

Maintaining homogenous field strength is not the only issue that magnet builders face in their quest to boost the signal. "Another challenge for all systems is that as you go up in field strength, you must address issues related to magnetic susceptibility," says Gordon. At higher field strengths, a magnetic field gradient can occur at the interface of materials with very different magnetic susceptibilities, such 
as tissue, bone and a void (in the sinuses). This can lead to artefacts in the images that must be accounted for during image analysis, says Gordon.

Currently, 3 T MRI systems are the standard for high-end human neuroimaging. But research-grade 7 T MRI instruments for human studies have come a long way in recent years with several companies, including Siemens Medical Solutions, GE Healthcare in Piscataway, New Jersey, and Philips in Andover, Massachusetts, now supplying second-generation versions of these systems. "The first generation of $7 \mathrm{~T}$ systems were monsters that needed 400 tonnes of shielding, but the second-generation systems are actively shielded so now in principle you don't need any iron to shield the magnet," says Gordon. But many researchers say that, even with the advances, $7 \mathrm{~T}$ systems still need more engineering work.

\section{The 'best' field strength?}

"The $7 \mathrm{~T}$ scanner is still a bit of a specialized device and, in my opinion, has yet to reach its full potential," says Larry Wald, director of the NMR core facility at the Athinoula A. Martinos Center for Biomedical Imaging in Charlestown, Massachusetts. Deshpande agrees: "There will be some ramp-up time with

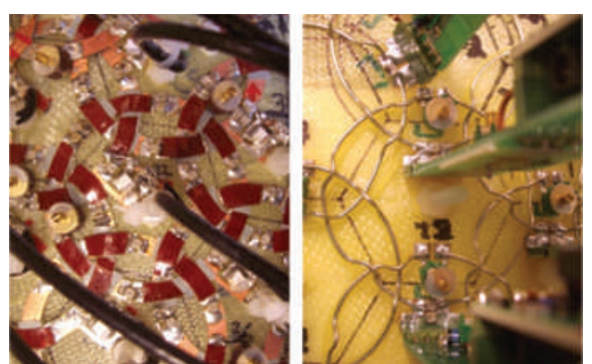

The radio-frequency coils used to transmit and detect MRI signals are being made more sensitive.

the $7 \mathrm{~T}$ in terms of applications and research. We need to find out how far we can push the instrument."

Whether $7 \mathrm{~T}$ will eventually become a clinically robust field strength for human MRI is not clear. "Ever since the beginning of MRI there has always been discussion about what field one should work at," says Koretsky. First there was the decision between using $0.5 \mathrm{~T}$ and $1.5 \mathrm{~T}$, and then between $3 \mathrm{~T}$ and $4 \mathrm{~T}$ magnets. Now, he says, a $7 \mathrm{~T}$ debate might start. "Some of the images from the brain at $7 \mathrm{~T}$ are truly amazing," says Deshpande. "We are seeing things that we have never seen at either $1.5 \mathrm{~T}$ or $3 \mathrm{~T}$."

Even as new $7 \mathrm{~T}$ human systems and $11.7 \mathrm{~T}$ and higher animal systems from companies such as Bruker Biospin and Varian in Palo Alto, California, expand in use within the neuroscience community, bigger magnets are being designed for cutting-edge animal and human imaging. In autumn 2008, the Martinos Center installed a $15 \mathrm{~T}$ magnet designed by Varian and Magnex Scientific in Walnut Creek, California, for imaging mice and rats, which Wald says is now at field strength and should be generating its first images in the coming weeks. The machine, used in conjunction with knockout mice with genes that have been 'turned off', should allow scientists to understand neural disease progression more effectively and even test potential drug therapies, says Wald.

Field strengths for human MRI magnets may reach double digits in the coming years. Both the NIH and NeuroSpin, a centre for ultra-highfield MRI in Saclay, France, have announced projects to construct 11.7 T MRI magnets for imaging human subjects. Although Koretsky says that it will be several years before these new magnets are installed and operational, he thinks the images will be worth the wait. "In the end you would love an anatomical picture that looks like a histological slice," he says, adding that these new magnets will get them closer than ever to making this a reality.

\section{CHANGING THE COLOUR OF MRI}

When Alan Koretsky, scientific director of the National Institute of Neurological Disorders and Stroke in Bethesda, Maryland, and Gary Zabow began to think about developing new contrast agents for magnetic resonance imaging (MRI), they took their design cues from the colourful world of molecular imaging. "It was looking at what existed and then figuring out some way to copy the idea of quantum dots," says Zabow, a physicist at the National Institute of Standards and Technology in Boulder, Colorado.

In molecular imaging, a quantum dot can generate a range of possible emission spectra simply by varying the size of the dot's inner core shell. This is a stark contrast to the traditional agents used in MRI, such as gadolinium or iron oxide, which are magnetic materials that alter the signal from the protons in the surrounding water, appearing as either darker or brighter spots on images. "It is sometimes difficult to tell the different agents apart from one another or from artefacts that make something brighter or darker," says Zabow. (At present, colour in MRI scans - such as those in this article - is assigned to shades of grey and added during processing.)

"One of the areas of MRI that has exploded over the past 5 or 6 years is the ability to track cells as they move around," says Koretsky. Although MRI cannot achieve single-cell resolution, a single cell can have sufficient magnetic-resonance contrast to be detected. But for researchers interested in tracking two or three cells at once, this level of differentiation is not enough.

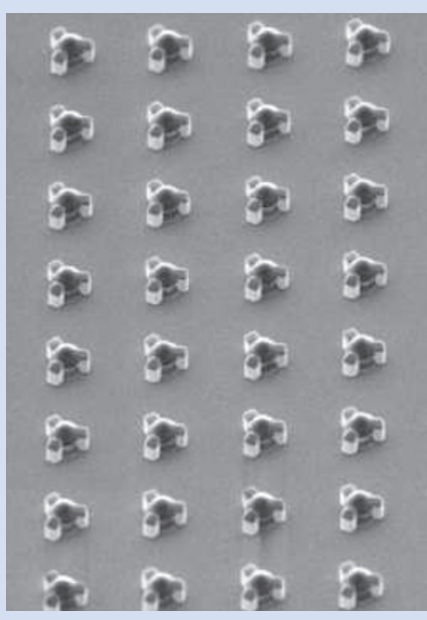

Electron-microscopy images of microfabricated contrast agents.
So Zabow and Koretsky microfabricated specific magnetic shapes that would create different magnetic fields and so shift the nuclear magnetic resonance frequency. "The existing magnetic particles do not shift the frequency - they just broaden it out," says Zabow. But the very precise shape of these new agents generates a corresponding precise frequency shift, similar to quantum dots, giving Zabow and Koretsky the possibility of creating different colours through different shapes and their specific frequency shifts.

The initial work consists of two discs with a gap between them in which the magnetic field can be generated. By varying the thickness or diameter of the discs, or the gap, different fields can be obtained so that when water passes between the discs the magnetic resonance of the water molecules flowing through the gap shifts (G. Zabow et al. Nature 453, 1058-1063; 2008).

Although the possible range of new colours is still to be determined, that is not the primary focus at the moment, says Zabow. They are working to improve the

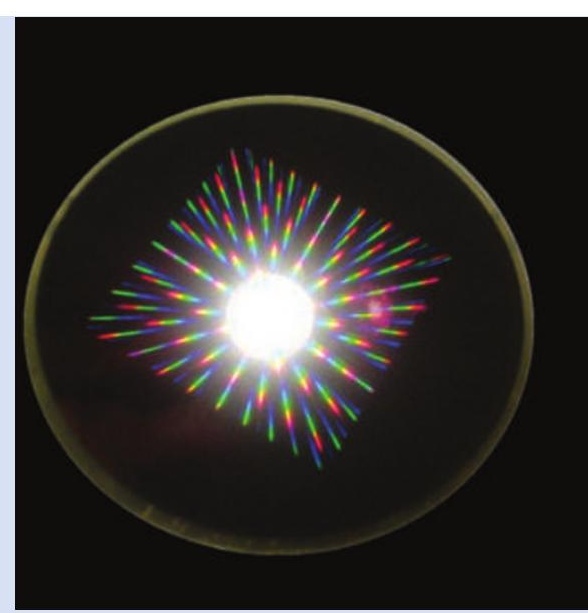

Microfabricated shapes provide potential for a wide range of possible colours when used in MRI.

fabrication process and make the magnetic particles smaller and more robust. "We are working first on that. The idea of having as many colours as possible falls out from there because in improving the fabrication we are getting the geometry more precise," he says.

Koretsky sees these new contrasting agents as adding a unique ability to MRI that no other radiological imaging technique possesses. 
High-strength magnets come with big technical challenges and hefty price tags. "These are demanding instruments. We can manufacture $16 \mathrm{~T}$ magnets for smallanimal imaging but they are exceptionally expensive," says Gordon. With a complete imaging system based on a $16 \mathrm{~T}$ magnet for small-animal imaging costing tens of millions of dollars and magnets for imaging humans often costing similar amounts, Gordon and others think that advances in radio-frequency-coil design could provide a more economical route to higher sensitivity.

"It is easy to forget, but if you dust off the old birdcage detectors that we used to use for the human head and compare those to what is shipping on a modern scanner these days - the sensitivity difference is pretty astonishing," says Wald. His group at the Martinos Center has been developing the coils for many years, but it might be the group's contributions to the development of parallel imaging that have had the biggest impact for MRI.

\section{Parallel evolution}

Radio-frequency coils are used as both transmitters to oscillate protons and as detectors to receive the signal. Ten years ago, single-channel coils were used for this purpose. But today, most instruments have multichannel coils, allowing the parallel acquisition of data during a scan. The benefits of parallel imaging can be seen in the 32-channel coil for head scans that Wald's group developed for brain imaging on Siemens' research-grade 7 T human MRI system. "We have a protocol that used to run as two 8.5-minute scans, but now we can do that in a single 3.5-minute scan," he says. Faster scans mean less time in the instrument for subjects, which presents new opportunities for the researchers at the larger imaging centres who routinely scan

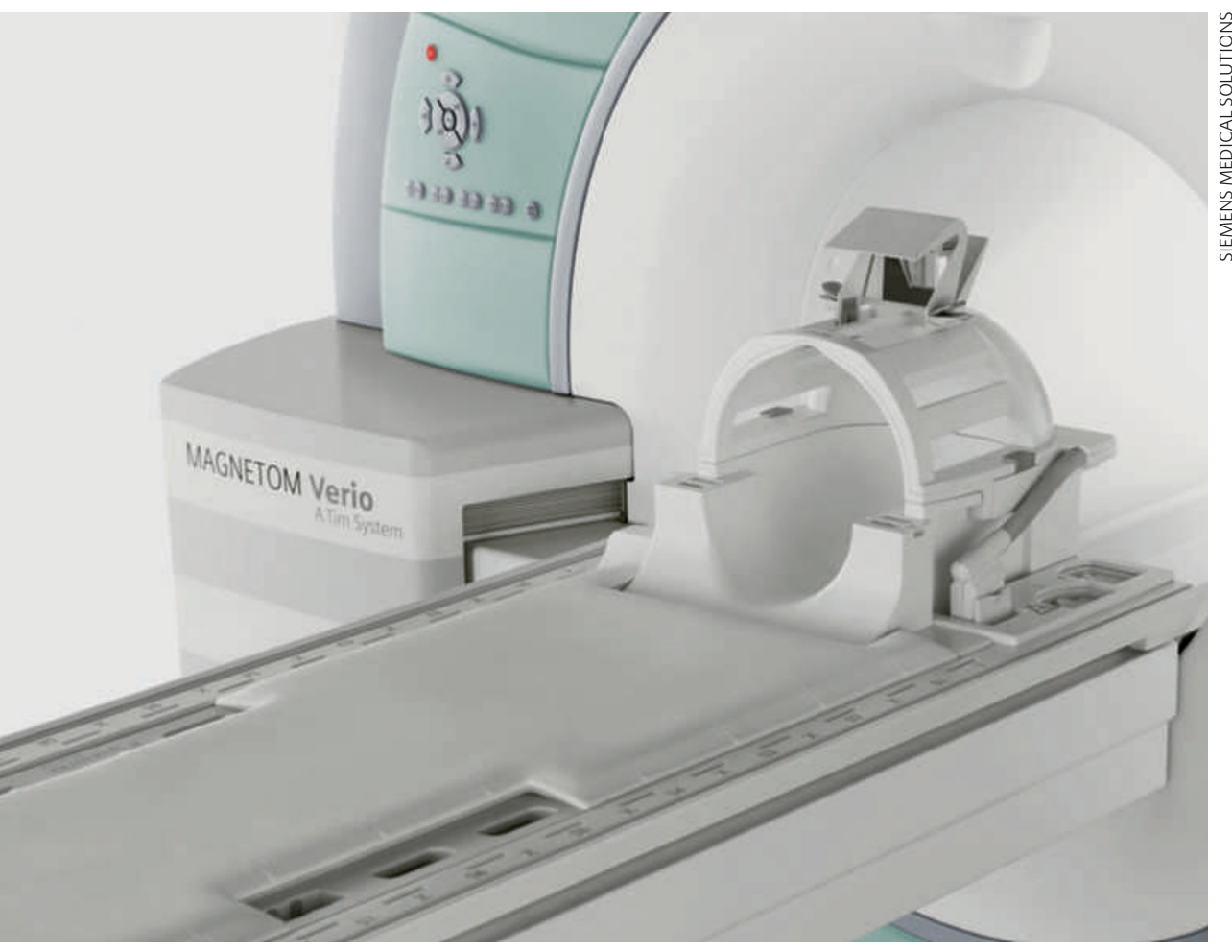

Siemens' MAGNETOM Verio 3 T scanner has a head and neck coil detection system. thousands of people a year. "There are now a lot of scientists interested in standardizing a fast morphometric protocol that would enable large-scale genotype/phenotype studies of neurological diseases," says Wald.

Wald's group has also worked at getting a better signal from its scans at lower field strengths with the development of a 96-channel head coil for use on Siemens' clinical 3 T MAGNETOM system. Although using more channels should mean better signal detection, this is not always the case. "In principle, if it is a 96-channel coil you can get a 96 factor acceleration in scan speed, but we do not come anywhere close to that," says Wald, a fact that is leading coil groups around the world to work on further

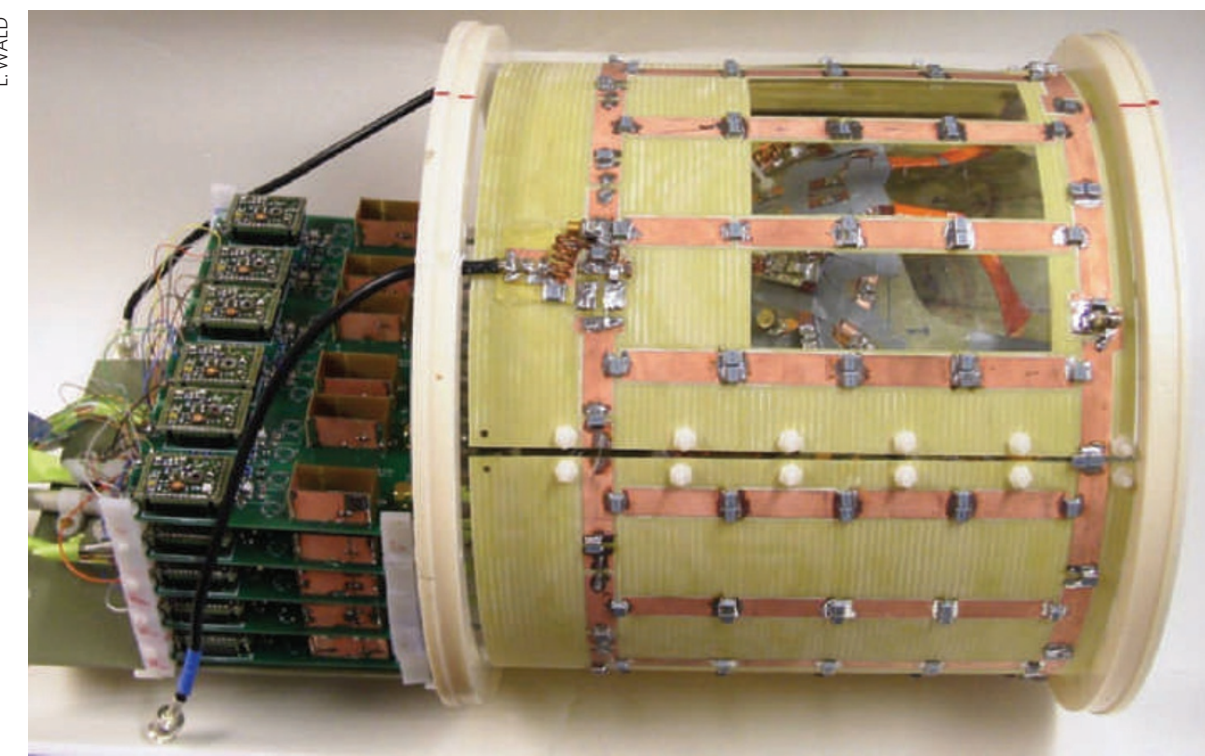

Multi-channel coils can reduce the time that patients need to stay in an MRI scanner. enhancing the sensitivity of multi-channel coils. The developments are leading to advances in commercially available coils. With its total imaging matrix (TIM) technology for scanning the whole body, Siemens offers multiple coils that can connect at the same time, allowing the system to decide on which of the coils to use depending on the section of body being scanned. In this way larger sections can initially be scanned at low resolution, followed by more focused higher-resolution scans of sections of interest, all without having to change the coil setups or move the patient out of the scanner to reposition the coil array. Philips now offers up to 33-element coils for neuroimaging on its $3 \mathrm{~T}$ Achieva TX system and GE Healthcare offers a series of coils designed for its Signa Infinity $1.5 \mathrm{~T}$ system.

\section{Divide and conquer}

As magnet field strengths increase, coil developers will have to keep improving sensitivity. "The detection side of it will continue to be hard, but we know what to do and how to do it now. The transmit side on the other hand is potentially most challenging," says Koretsky.

For radio-frequency transmitting, the technical challenges increase with field strength because the human body starts to distort the radio frequency at high field strengths affecting the radio-frequency transmission. Some progress has been made on this front already, with researchers working on the idea of parallel transmission using multiple radiofrequency transmitters that are simultaneously excited. By using parallel transmission it is possible to eliminate the homogeneity problems caused by the shorter wavelengths generated 
at higher frequencies. "Parallel transmit breaks it down into chunks, which can be put back together to get a uniform transmit in the end," says Wald. "A sort of 'divide and conquer' approach helps to get around this."

But another recent development might further change how transmission is done. In February 2009, Klaas Pruessmann's group at the Institute for Biomedical Engineering in Zurich, Switzerland, described a new transmission and detection technique called travelling-wave nuclear magnetic resonance (D. O. Brunner et al. Nature 457, 994-998; 2009). In this approach, the radiofrequency wave travels down the bore of the magnet, which acts like a waveguide. Although this means that the bore has to be big enough
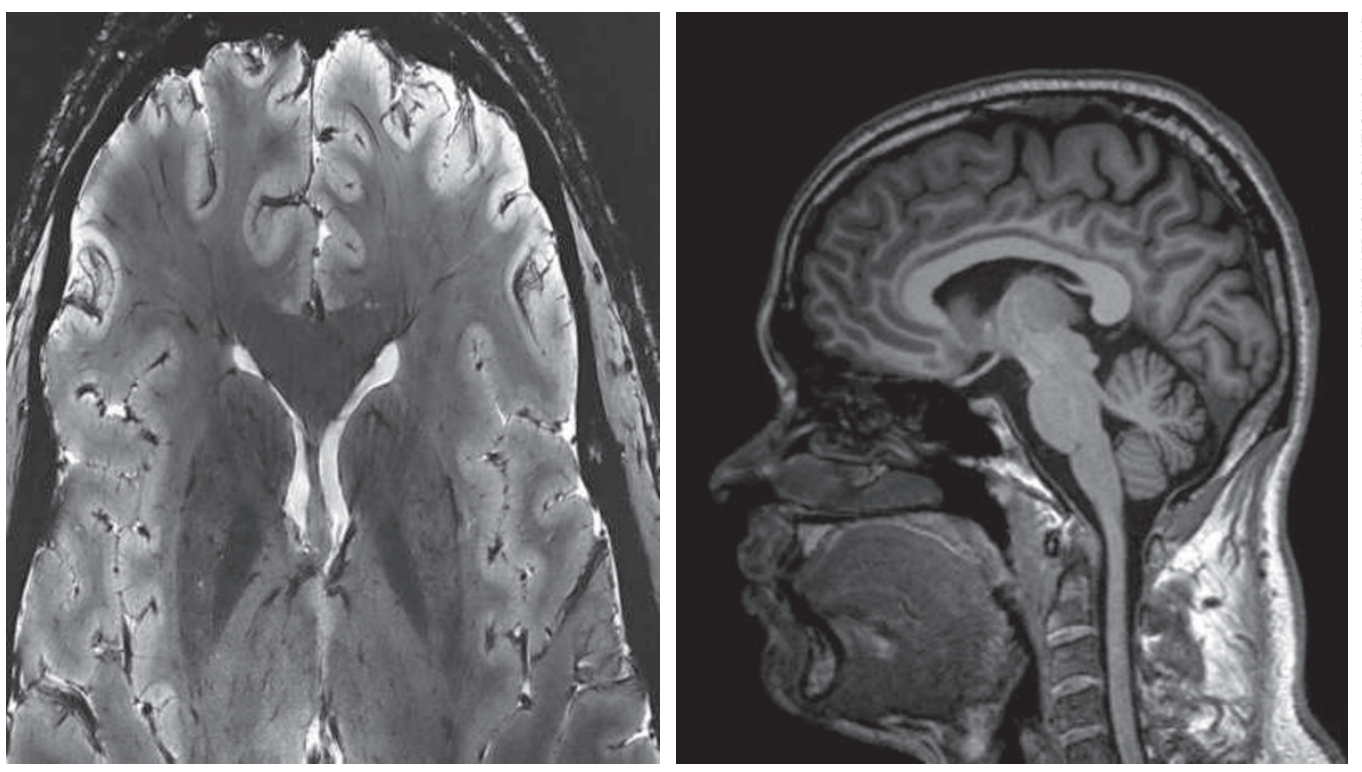

Images of the human brain taken with 7-tesla MRI scanners.

compared with the wavelength for the wave to travel, many researchers are encouraged by the potential of this approach to solve some of the radio-frequency transmission issues.

"The most exciting thing with the travelling wave approach is that you not only get the apparatus for transmit out of the bore of the magnet, but it also offers the potential to have a more homogenous excitation," says Wald. Although the approach will only work for 7 T-and-higher strength systems, Wald says that this is where this type of technology is most needed, given the transmission problem and the decreasing bore sizes that come with larger magnets.

\section{Adding function to the form}

Signal in MRI is much like currency, says Deshpande. By boosting the signal with stronger magnets and multi-channel coils, researchers can "spread their currency around to get shorter scan times and higher

\section{OPTIMUM SIGNAL-TO-NOISE RATIO}
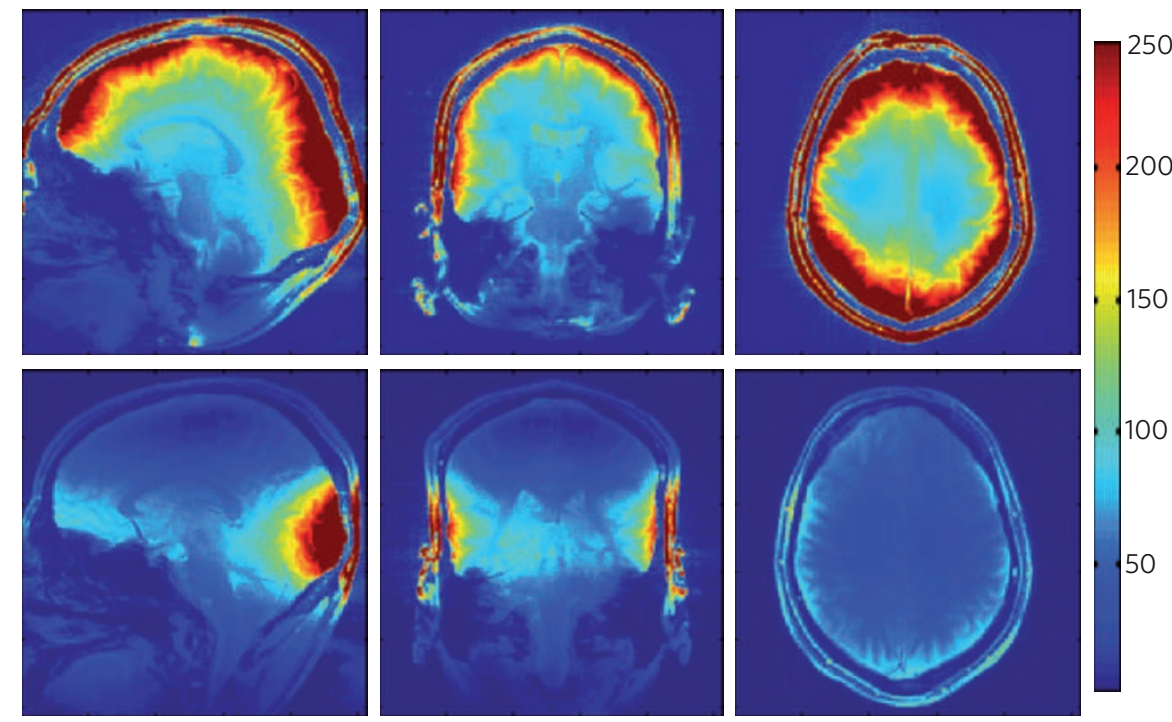

The MRI signal with a 32-channel coil array (top row) is clearer than with an 8-channel array (bottom row).

resolution when doing functional imaging". Functional MRI (fMRI) is a technique that ooks for changes in the levels of deoxyhaemo' an indicator of blood flow in the brain due to neural activity. By observing neural activity in subjects performing different tasks, $\mathrm{fMRI}$ is helping cognitive neuroscientists gain a better handle on how the brain works. "It is that hrough the melding of behaviour sciexperimental paradigms along with experiments that grows the field," says Hirsch.

Although the field continues to grow through technology development and improving experimental design, mainstream surgeons are using the technique to map patients' brains before surgery, but at the moment this is the only application of fMRI for which physicians can be reimbursed through insurance companies in the United States.

But this is not stopping researchers from moving forwards with new fMRI studies. Omneuron, a life-sciences company in Menlo Park, California, is currently recruiting volunteers for a clinical trial on the neural basis of chronic pain. Using fMRI, researchers at Omneuron are mapping the brain activities of participants performing specific mental tasks. Other companies, such as the Applied fMRI Institute in San Diego, California, with its 3 T Siemens Tim MAGNETOM Trio scanner, provide both fMRI and anatomical MRI services, and a host of companies, including Compumedics Neuroscan in Charlotte, North Carolina, Visage Imaging in Andover, Massachusetts, and Prism Clinical Imaging in Wauwatosa, Wisconsin, are providing software to integrate the functional information of fMRI with other modalities including EEG and PET.

The biggest changes in fMRI might come as the technology becomes more personalized. "One of the signs of brain imaging advancement is how well can we apply the benefits of neuroimaging to personalized patient care," Hirsch says. But even more sensitivity and accuracy will probably be required for this, because fMRI studies often rely on multiple subjects.

By improving understanding of why we make certain decisions and how our brains differ in shape, MRI is changing the way we view the brain. And many researchers think more insights are on the way as the technology continues to advance. "MRI is going to move from a tool for identification to one that helps us understand mechanisms," says Koretsky. "From being able to say there is a tumour there to understanding how that tumour formed and grew."

Nathan Blow is the technology editor for Nature and Nature Methods. 